\title{
Minimally Invasive Resection of Deep-seated High-grade Gliomas Using Tubular Retractors and Exoscopic Visualization
}

\author{
Rajiv lyer $^{1}$ Kaisorn L. Chaichana ${ }^{2}$ \\ ${ }^{1}$ Department of Neurosurgery, Johns Hopkins University, Baltimore, \\ Maryland, United States \\ 2 Mayo Clinic, Jacksonville, FL, United States
}

Address for correspondence Kaisorn L. Chaichana, MD, Department of Neurosurgery, Mayo Clinic, 4500 San Pablo Road S, Jacksonville, FL 32224 (e-mail: chaichana.kaisorn@mayo.edu).

J Neurol Surg A 2018;79:330-336.

\begin{abstract}
Background and Study Aims/Objective Deep-seated high-grade gliomas (HGGs) represent a unique surgical challenge because they reside deep to critical cortical and subcortical structures and infiltrate functional areas of the brain. Therefore, accessing and resecting these tumors can often be challenging and associated with significant morbidity. We describe the use of minimally invasive approaches to access deep-seated HGGs to achieve extensive resections while minimizing surgical morbidity. Materials and Methods All patients who underwent resection of a deep-seated intraparenchymal HGG with the use of a tubular retractor with exoscopic visualization from January 2016 to May 2017 were identified prospectively at a single institution. Variables evaluated included tumor location, pre- and postoperative neurologic function, extent of resection, and length of hospital stay.

Results Overall, 14 patients underwent resection of an HGG (11 glioblastomas, 3 anaplastic astrocytomas) with a tubular retractor under exoscopic visualization. Seven tumors (50\%) involved the thalamus, three (21\%) the motor corticospinal tract, two (14\%) the inferior frontal occipital fasciculus, one (7\%) each the basal ganglia and optic pathway. The median preoperative Karnofsky Performance Score (KPS) was 70 (interquartile range: 55-80), where the major presenting symptom was motor weakness in seven (50\%). The

Keywords

- brain path

- deep-seated

- exoscope

- glioblastoma

- minimally invasive average plus or minus the standard error of the mean percentage resection was $97.0 \pm 1.2 \%$. The median hospital stay was 4 days (range: $2-7$ ). At 1 month postoperatively, median postoperative KPS (within 30 days) was 87 (range: $77-90$ ), where eight (57\%) were improved, five (36\%) were stable, and one $(7 \%)$ was worse postoperatively.

Conclusions Deep-seated HGGs can be accessed, visualized, and resected using tubular retractors and exoscopic visualization with minimal morbidity.
\end{abstract}

\section{Introduction}

The general treatment paradigm for high-grade gliomas (HGGs) involves maximal resection followed by adjuvant chemoradiation. $^{1-4}$ This portends a survival benefit for patients harboring this aggressive disease, especially when critical resection thresholds are achieved while avoiding

received

August 21, 2017

accepted after revision

January 10, 2018

published online

April 23, 2018

iatrogenic deficits. ${ }^{1-4}$ Patients who develop deficits have worsened survival independent of extent of resection. ${ }^{5}$ Patients with deep-seated HGGs in locations such as the basal ganglia and thalamus have arguably the highest surgical risk of developing neurologic deficits. ${ }^{5,6}$ Although the management strategy of extensive resection for more superficially located HGGs is widely accepted, the treatment of

(C) 2018 Georg Thieme Verlag KG Stuttgart · New York
DOI https://doi.org/ 10.1055/s-0038-1641738. ISSN 2193-6315. 
deep-seated HGGs is less clear. ${ }^{7,8}$ Some clinicians opt to perform stereotactic needle biopsies to minimize surgical morbidity; others perform open resections with significant risk of iatrogenic deficits. ${ }^{7,8}$ Aggressive surgical resection of these lesions, however, may be beneficial for patients as long as deficits are avoided. ${ }^{9-11}$ These deficits can occur when accessing the tumor, which may require significant brain resection, disruption, and/or retraction, as well as during tumor resection, because these lesions are located in and around eloquent regions. ${ }^{10-12}$

Improved outcomes for patients with deep-seated HGGs could be achieved if these lesions can be accessed and resected without endangering surrounding cortical and subcortical structures. Minimally invasive techniques, namely tubular retractors, were developed to access deep-seated intracranial hemorrhages and intraventricular tumors. ${ }^{12-14}$ These retractors are typically placed within a sulcus and splay white matter tracts to approach subcortical locations with minimal morbidity. ${ }^{12-14}$ They provide a protected corridor for accessing and potentially resecting these hardto-reach lesions. ${ }^{12-14}$ Although the removal of clots and intraventricular lesions is inherently different than intraparenchymal tumor, ${ }^{15-17}$ the efficacy of these techniques for nonbiopsy surgical resection of deep-seated HGGs remains less clear. In this study, we evaluated our results utilizing these tubular retractors for the resection of deep-seated HGGs with exoscopic visualization.

\section{Materials and Methods}

Before the start of this study, institutional review board approval was obtained to collect patient data prospectively. Formal patient consent was not required because all the information was tabulated in a blinded fashion. There was no identifying patient information.

\section{Patient Selection}

All patients operated on for a deep-seated HGG from January 2016 to May 2017 at a single academic tertiary care institution using tubular retractors with exoscopic visualization were identified prospectively and included in this case series (-Figs. 1-3). All patients were operated on by the senior author (K.L.C.). A deep-seated location was defined as any subcortical location in close proximity to the basal ganglia and thalamus including white matter tracts based on diffusion tensor imaging (DTI). Tumors within the ventricles were excluded. Variables collected prospectively included age, sex, tumor location, pre- and postoperative
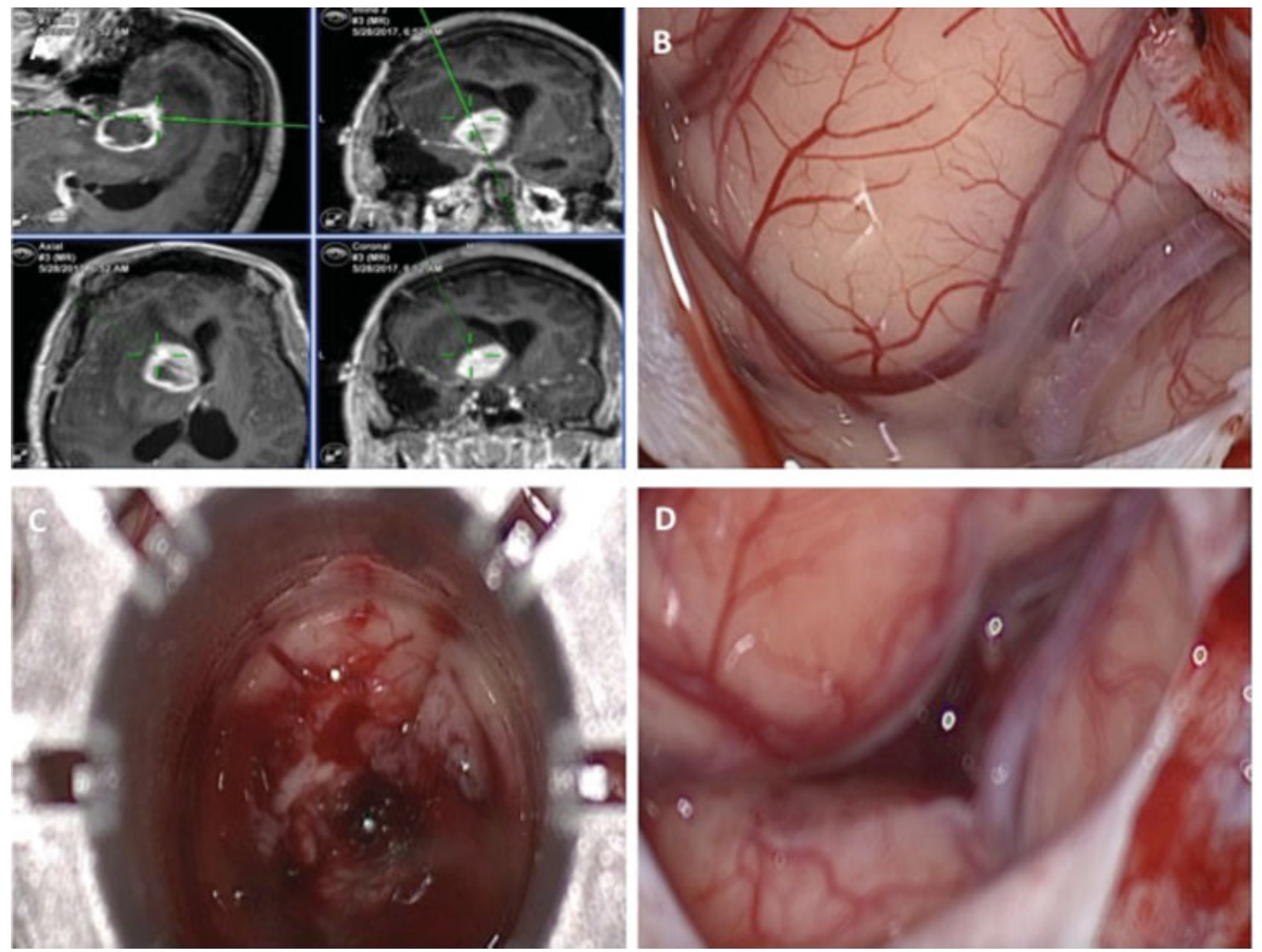

Fig. 1 Intraoperative image of a right thalamic glioblastoma multiforme patient who underwent resection with a tubular retractor under exoscopic visualization. (a) Intraoperative navigation images showing targeting of thalamic lesion. (b) Exoscopic image of desired sulcus to be targeted for accessing the lesion. (c) Sulcus is opened under exoscopic visualization and a tubular retractor is placed through the sulcus to expose the lesion. (d) After tumor is removed, retractor is removed, and sulcus with sulcal vessels is preserved. 

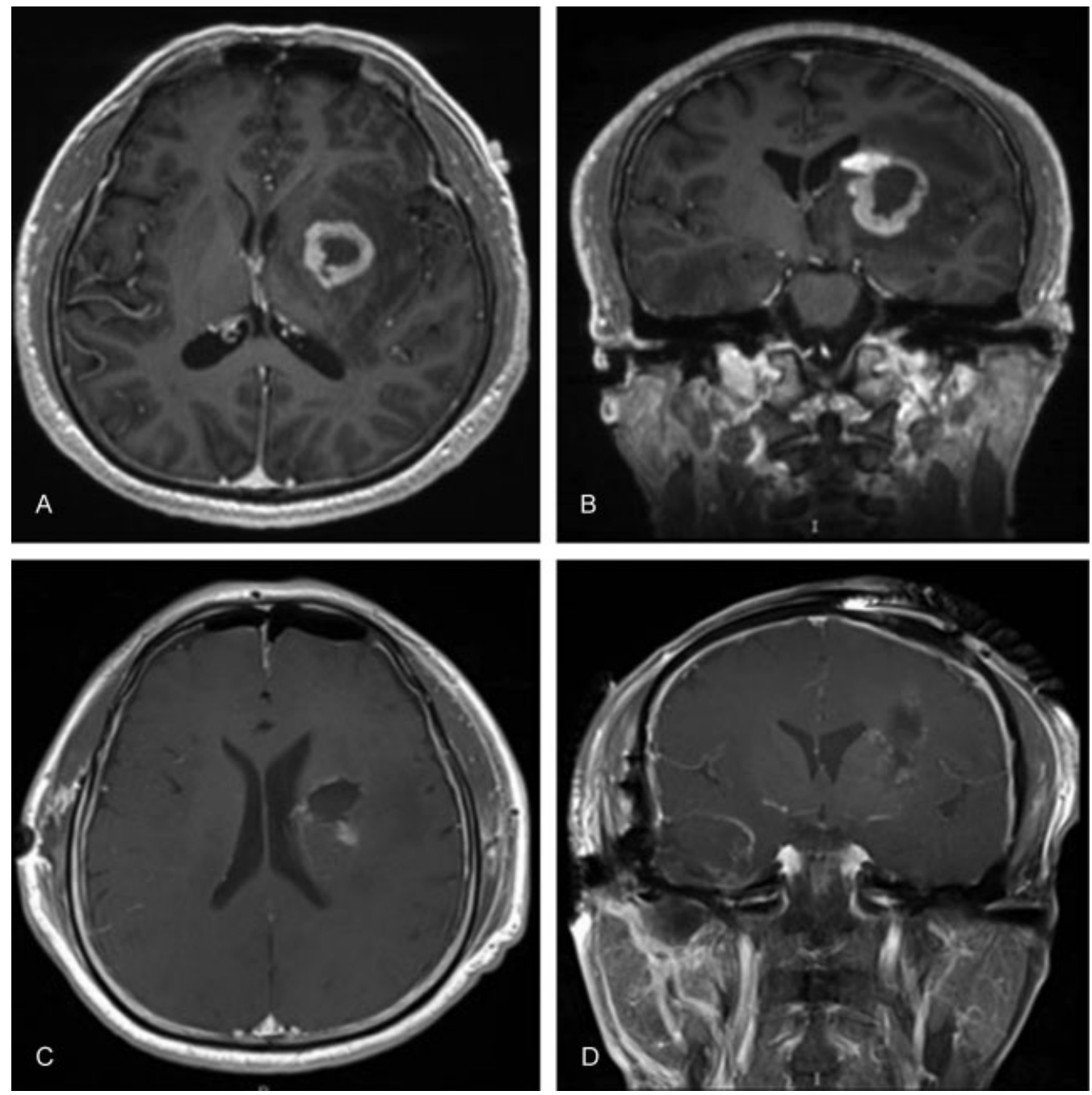

Fig. 2 Example of a patient with a left basal ganglia glioblastoma multiforme who underwent resection with a tubular retractor. (a, b) Preoperative axial and coronal T1-weighted magnetic resonance imaging (MRI) with gadolinium demonstrating a left basal ganglia tumor. (c, d) Postoperative axial and coronal T1-weighted MRI with gadolinium demonstrating gross total resection of the tumor.

neurologic function, and functional status (Karnofsky Performance Score [KPS]), pre- and postoperative tumor volume, length of hospital stay, and duration of follow-up. KPS scores were recorded immediately preoperatively, upon discharge, and at subsequent follow-up visits.

\section{General Treatment Strategy}

Patients who presented with deep-seated lesions causing significant mass effect were considered for nonbiopsy surgical resection. The goal of each case was to achieve maximal resection without causing a significant or progressive neurologic deficit. In each case, it was determined that stereotactic needle biopsies would have left significant residual tumor with persistent mass effect. A tubular retractor was chosen when there was concern for morbidity related to tumor exposure through traditional means and when the tumor was deep to and/or involved in eloquent cortical and/ or subcortical regions (i.e., motor cortex, motor corticospinal tracts, etc.). ${ }^{12}$ Patients underwent magnetic resonance imaging (MRI) with and without gadolinium 1 to 2 days before the surgery. In most cases, DTI was performed to help delineate critical white matter tracts. The trajectory toward the lesion was planned preoperatively and was chosen based on minimizing the potential morbidity associated with accessing the lesion (-Fig. 1a). In general, a trans-sulcal route was chosen to minimize damage to critical cortical regions and white matter tracts ( $\mathbf{- F i g .} \mathbf{1 b}$ ). Resection was aimed at the contrast-enhancing portion of the lesion and not fluid-attenuated inversion recovery changes. Intraoperative somatosensory evoked potential, motor evoked potential, and electroencephalogram monitoring was used in all cases.

Based on intraoperative navigation and surgical planning, a 3-cm skin incision was made overlying the planned trajectory. A $\sim 2$-cm craniotomy was performed, and the dura was then opened in a cruciate fashion. A 0 - or 90-degree exoscope (Vitom, Karl Storz, El Segundo, California, United States) attached to a pneumatic arm (UniArm, Mitaka, Park City, Utah, United States) was used to provide visualization and magnification through the tubular retractor. This exoscopic 

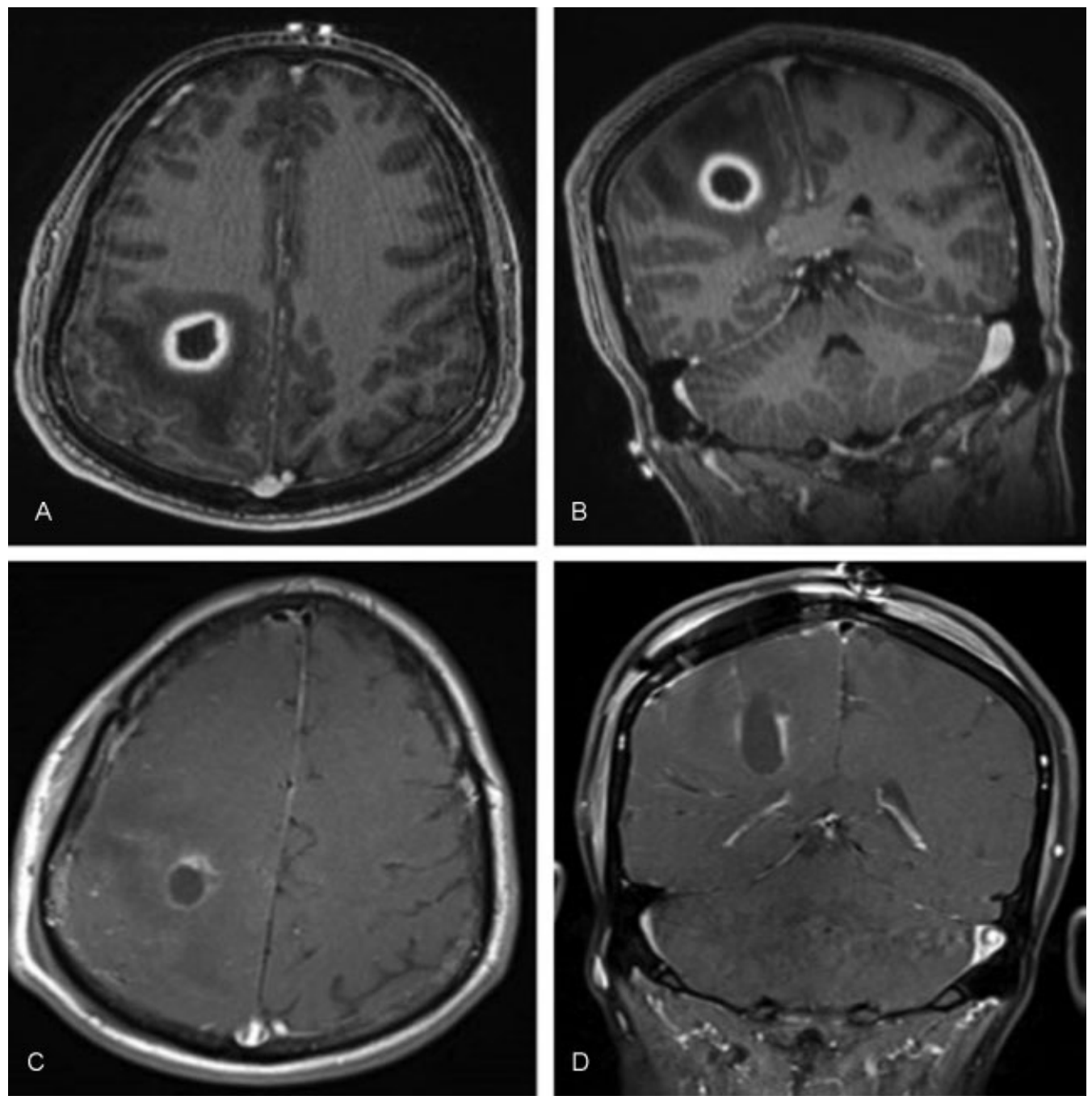

Fig. 3 Example of a patient with a right peri-Rolandic corticospinal glioblastoma multiforme (GBM) resected with a tubular retractor. (a, b) Preoperative axial and coronal T1-weighted magnetic resonance imaging (MRI) with gadolinium demonstrating a right peri-Rolandic GBM involving motor corticospinal tracts. (c, d) Postoperative axial and coronal T1-weighted MRI with gadolinium demonstrating gross total resection of the tumor.

visualization involves the use of an endoscope that is external to the body and hovers over the surgical field. ${ }^{12}$ Under exoscopic visualization, the preoperatively identified sulcus is opened to its depth. The preselected tubular retractor (BrainPath, Nico Corp., Indianapolis, Indiana, United States) of the desired length was passed through the sulcus under frameless stereotactic guidance to reach the most superficial component of the tumor ( - Fig. 1c). Along this trajectory, the inner cannula was removed, and an ultrasound probe (Hitachi Aloka, Wallingford, Connecticut, United States) was placed to confirm appropriate trajectory and lesion localization. Once the superficial surface of the lesion was reached, the retractor was fixated with a Leyla retractor system (B. Braun Medical Inc., Bethlehem, Pennsylvania, United States).

The tumor was then debulked and resected using primarily suction, a tissue-biting device (Myriad, Nico, Indianapolis, Indiana, United States), and bipolar cautery. For larger lesions, the Leyla retractor was loosened and the tubular retractor rotated and swiveled to facilitate resection of tumor beyond the boundaries of the retractor. After resection, the retractor was slowly withdrawn ( - Fig. 1d). Closure of the dura, bone, and skin were completed in standard fashion. Postoperative MRI was obtained 1 to 2 days after surgery, and the volumetric extent of resection was assessed as previously described based on the contrast-enhanced portion. ${ }^{2,18}$

\section{Results}

Fourteen patients underwent resection of an HGG (11 [79\%] glioblastoma multiforme, 3 [21\%] anaplastic astrocytoma) with a tubular retractor under exoscopic visualization during the reviewed period ( - Table 1 ). Ten $(71 \%)$ were male and four $(29 \%)$ were female, with an average age plus or minus standard deviation of $42.2 \pm 16.7$ years. The location of the epicenter of the tumor was the thalamus in seven (50\%), motor corticospinal tract in three (21\%), inferior frontal occipital fasciculus (IFOF) in two (14\%), basal ganglia in 
Table 1 Patients who underwent resection of a high-grade glioma located in deep-seated regions using tubular retractors with exoscopic visualization

\begin{tabular}{|c|c|c|c|c|c|c|}
\hline Age, $y$ & Sex & Location & Presenting symptoms & $\begin{array}{l}\text { Preoperative } \\
\text { tumor } \\
\text { volume, } \mathrm{cm}^{3}\end{array}$ & $\begin{array}{l}\text { Percentage } \\
\text { resection }\end{array}$ & $\begin{array}{l}\text { Preoperative versus } \\
\text { postoperative KPS }\end{array}$ \\
\hline 51 & $\mathrm{M}$ & Thalamus & Hemiparesis & 6.7 & 100 & Stable \\
\hline 32 & M & Corticospinal tracts & Hemiparesis & 3.0 & 100 & Improved \\
\hline 54 & M & IFOF & Seizures & 15.0 & 100 & Stable \\
\hline 68 & M & Optic pathway & Visual field loss & 2.9 & 100 & Stable \\
\hline 73 & M & Corticospinal tracts & Leg weakness & 1.2 & 90 & Improved \\
\hline 18 & $\mathrm{~F}$ & Thalamus $^{\mathrm{a}}$ & Hemiparesis & 16.3 & 97 & Improved \\
\hline 20 & $\mathrm{~F}$ & Thalamus & Headaches, vision loss & 23.4 & 99 & Improved \\
\hline 50 & M & IFOF & Aphasia & 4.8 & 100 & Stable \\
\hline 36 & M & Thalamus $^{\mathrm{a}}$ & Obtundation & 18.6 & 97 & Improved \\
\hline 51 & $\mathrm{M}$ & Thalamus & $\begin{array}{l}\text { Headaches, hemipar- } \\
\text { esis, vision loss }\end{array}$ & 23.8 & 93 & Worsened \\
\hline 46 & $\mathrm{M}$ & Corticospinal tracts & Seizures & 1.8 & 100 & Stable \\
\hline 26 & $\mathrm{M}$ & Thalamus $^{\mathrm{a}}$ & Hemiparesis & 25.9 & 85 & Improved \\
\hline 33 & $\mathrm{~F}$ & Thalamus & Hemiparesis & 12.7 & 95 & Improved \\
\hline 33 & $\mathrm{~F}$ & Basal ganglia & Arm weakness & 8.1 & 100 & Improved \\
\hline
\end{tabular}

Abbreviations: IFOF, inferior frontal occipital fasciculus; KPS, Karnofsky Performance Score.

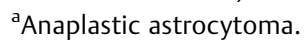

one (7\%), and optic pathway in one (7\%). The median preoperative tumor volume was $10.4 \mathrm{~cm}^{3}$ (interquartile range: 3.5-18.0). The median preoperative KPS was 70 (range: 5580 ), and the major presenting symptoms were motor weakness in seven (50\%; four with upper and lower extremity hemiparesis), headaches/nausea/vomiting in four (29\%), vision loss in two (14\%), and seizures in one (7\%).

Following surgery, the median tumor volume was $0.1 \mathrm{~cm}^{3}$ (range: 0-0.5), where gross total resection was achieved in seven (50\%). The average plus or minus standard error of the mean percentage resection was $97.0 \pm 1.2 \%$. No patients had significant hemorrhage. At 1 month postoperatively, median postoperative KPS (within 30 days) was 87 (range: 77-90), where eight (57\%) were improved, five (36\%) were stable, and one $(7 \%)$ was worse postoperatively compared with preoperative function. Of the seven patients with preoperative motor weakness, five patients had improved postoperative strength, one had stable antigravity arm weakness, and one worsened upper extremity weakness. The one patient who worsened had a preoperative KPS of 60 and declined to 50 at 1 month postoperatively with increased upper extremity weakness from 4 of 5 to 2 of 5 , and postoperative imaging revealed no significant hemorrhage or stroke. This progressive weakness was likely due to surgical manipulation of the posterior limb of the internal capsule and progressive infiltration of the tumor. Of the four patients with signs of increased intracranial pressure, symptoms of headaches/nausea/vomiting resolved. Of the two patients with vision loss, the visual field deficit remained stable in one and improved but was still present in another. The patient with preoperative seizures remained seizure free but on antiepileptics. The median hospital stay was 4 days (range: $2-7$ ), with eight patients (57\%) discharged to home. No patients incurred postoperative medical complications including infection, pneumonia, deep vein thrombosis/ pulmonary embolism, sepsis, and/or seizures. The median follow-up duration was 3.5 months (range: 1.2-6.8) after surgery.

\section{Discussion}

In this study, 14 patients underwent nonbiopsy surgical resection of a deep-seated HGG using minimally invasive techniques with tubular-assisted resection and exoscopic visualization. The locations of these lesions included the thalamus, motor corticospinal tract, IFOF, basal ganglia, and optic pathway. The average percentage resection was $97.0 \%$, and at 30 days following surgery eight (57\%), five (36\%), and one (7\%) patient had improved, stable, and worse KPS status as compared with preoperatively. Minimally invasive techniques can therefore be used to access and resect HGG in arguably the most critical locations.

HGGs remain a major neurosurgical challenge due to their poor prognosis. ${ }^{1-4}$ In recent years, several studies have demonstrated a survival benefit for HGGs when critical resection thresholds are achieved, and advocate the need for developing techniques and apparatuses to facilitate extensive resection of these tumors while minimizing injury to normal surrounding structures. ${ }^{2,4,19,20}$ These studies were primarily limited to tumors that are more amenable to resections, namely superficial and noneloquent tumors. ${ }^{2,4,19,20}$ Deep- 
seated tumors are challenging because they reside below and within eloquent cortical and subcortical structures. ${ }^{10,11}$ Surgical morbidity can occur during accessing the tumor, as well as during resection, likely as a result of manipulating and potentially damaging critical gray matter structure, white tract fibers, and/or small vessel perforators that are especially concentrated in these regions. ${ }^{10,11}$ Moreover, these patients tend to have worsened preoperative neurologic status. ${ }^{10,11}$

Because of this high risk of iatrogenic injury, many surgeons elect to perform stereotactic needle biopsies of such lesions to establish a diagnosis, relying on adjuvant chemoradiation for tumor-killing effects. ${ }^{8,10,11}$ Kelly described 72 patients with thalamic astrocytomas (from grade 1 to 4 ), where 50 patients underwent stereotactic needle biopsy and 22 underwent resection. ${ }^{11}$ of the 50 patients who underwent needle biopsy, 6 (12\%) were worsened by the procedure, and $3(6 \%)$ died..$^{11}$ of the 22 patients who underwent resection, 5 (23\%) were worsened by the procedure, and 1 (5\%) died. ${ }^{11}$ Extent of resection was not analyzed. ${ }^{11}$ Cao et al studied 111 patients with thalamic gliomas (50 HGGs and 61 low-grade gliomas). ${ }^{10}$ Using standard techniques, gross total resection was achieved in 29 (26.1\%), subtotal resection in 54 (48.6\%), and partial resection in $21(18.9 \%) .{ }^{10}$ Postoperatively, 23 patients $(21.7 \%)$ had worsened motor function, 6 (5\%) required operative decompression for intracranial hypertension, vision deficit in 6 (5\%), and mortality in 5 (4.5\%). ${ }^{10}$ These deep-seated tumors therefore have a significant risk regardless of approach. ${ }^{10,11}$

Numerous methods have been developed to enable neurosurgeons to achieve maximal resection of HGGs more safely and effectively including frameless stereotaxy with neuronavigation, awake craniotomy with intraoperative cortical and subcortical brain mapping, fluorescence-guided tumor resection, intraoperative imaging, and others. ${ }^{21-26}$ These methods, however, are limited for deep-seated lesions because these lesions are deep to and involve eloquent brain regions. The traditional approach for these lesions is either a stereotactic needle biopsy or open craniotomy. The needle biopsy is the least invasive; however, only a limited resection can occur with this method. Therefore, there is significant residual tumor and mass effect, lack of tissue for molecular analyses and banking, and it still carries significant risks. ${ }^{10,11}$ Traditional open intracranial approaches often require some degree of brain manipulation through fixed or dynamic retraction, as well as possible brain resection, which contributes to surgical morbidity. ${ }^{10,11,27}$

To combat these effects when tackling deep-seated intracranial lesions, several iterations of fiber-sparing tubular retractor systems have been developed to provide safe surgical corridors for accessing and resecting deep-seated lesions. ${ }^{15,17,28-31}$ In addition, visualization is difficult because adequate light is needed to reach the tumor. ${ }^{12}$ Exoscopes help provide adequate lighting, greater degree of freedom, larger focal point ranges, and more maneuverability than typical surgical microscopes. ${ }^{12}$ As a result, tubular retractor systems and exoscopes have garnered much interest in the neurosurgical community for the evacuation of deep intracerebral hemorrhages, as well as the resection of intraventricular and periventricular cysts and tumors. ${ }^{14,15}$ The ability to resect deep-seated HGGs safely using minimally invasive techniques is less clear.

In this study, we successfully utilized fiber-sparing tubular retractors for the resection of deep-seated supratentorial HGGs with exoscopic visualization. Use of this technology allowed us to perform small preplanned craniotomies that enabled accurate triangulation of the target lesions. Preoperative DTI sequences aided in the selection of these surgical trajectories that were generally tailored to avoid crossing white matter tracts orthogonally. The trans-sulcal approach was most commonly used for retractor passage. It prevented the need for a corticectomy in most cases and thus minimized brain manipulation and resection. Neuronavigation and intraoperative ultrasound were used to visualize the path of the retractor through parenchyma in real time, which allowed us to center the retractor carefully at the outer edge of the target lesion at the start of resection. Often working down the barrel of a deep corridor, tumor resection was possible with the use of specialized tissue-biting devices that did not block exoscopic visualization. This allowed us to achieve excellent volumetric HGG resections, on average $>95 \%$. Larger lesions required retractor adjustment to see the poles of the tumor, an important consideration during craniotomy planning to avoid retractor motion limitation by the bone edge. Functionally, most patients in our study fared well, with an improved or stable KPS in $>93 \%$.

This study is the first to describe the efficacy of using minimally invasive approaches for the resection of deepseated HGGs. It provides an alternative to stereotactic needle biopsies and traditional open approaches, where the risks are minimized by using tubular retractors to splay white matter tracts and provide a protected corridor for resection. This method allows extensive resection while minimizing possible morbidity from iatrogenic injury.

However, our study is not without limitations. This study was not designed to evaluate if this technique and/or extensive resection was associated with prolonged survival or delayed recurrences. It was meant to serve as a proof of principle to demonstrate that these techniques can be used for these high-risk deep-seated HGGs, namely those in the thalamus and basal ganglia where needle biopsies are historically the treatment of choice in this region given the high surgical risk. Randomized studies comparing techniques and studies with longer-term follow-up would be needed to evaluate survival outcomes.

Our small sample size also limits detailed statistical analysis and generalizability of the technique. Larger studies would be needed to verify these results. Moreover, our study was limited to intraparenchymal high-grade tumors. We excluded low-grade gliomas and intraventricular tumors that are surgically distinct from intraparenchymal highgrade tumors, and therefore cannot be generalized to these other tumor types and locations. Lastly, our short follow-up period also limits discussion of possible long-term effects and neurologic deficits. Nonetheless, we believe this technique provides a successful way to perform resections on deepseated HGGs that are typically reserved for needle biopsies. Despite these limitations, we believe our study highlights a 
technique by which tubular retractors can be used to resect deep-seated HGGs safely and effectively.

\section{Conclusion}

An increasing number of studies have documented improved patient outcomes with extensive resection of HGGs. Deepseated HGGs associated with the basal ganglia, thalamus, and critical white matter tracts can be surgically challenging to access and to resect without neurologic injury. As a result, many surgeons favor needle biopsies of these tumors to establish a diagnosis alone that does little to improve mass effect and to change the natural history of the disease. We provide a minimally invasive solution to this problem, utilizing tubular retractors with exoscopic visualization to access and extensively resect deep-seated HGGs with minimal resultant morbidity.

\section{Conflicts of Interest}

Kaisorn L. Chaichana is a course educator for Nico BrainPath.

\section{References}

1 Stupp R, Mason WP, van den Bent MJ, et al; European Organisation for Research and Treatment of Cancer Brain Tumor and Radiotherapy Groups; National Cancer Institute of Canada Clinical Trials Group. Radiotherapy plus concomitant and adjuvant temozolomide for glioblastoma. N Engl J Med 2005;352(10):987-996

2 Chaichana KL, Jusue-Torres I, Navarro-Ramirez R, et al. Establishing percent resection and residual volume thresholds affecting survival and recurrence for patients with newly diagnosed intracranial glioblastoma. Neuro Oncol 2014;16(01):113-122

3 Lacroix M, Abi-Said D, Fourney DR, et al. A multivariate analysis of 416 patients with glioblastoma multiforme: prognosis, extent of resection, and survival. J Neurosurg 2001;95(02):190-198

4 Sanai N, Polley MY, McDermott MW, Parsa AT, Berger MS. An extent of resection threshold for newly diagnosed glioblastomas. J Neurosurg 2011;115(01):3-8

5 McGirt MJ, Mukherjee D, Chaichana KL, Than KD, Weingart JD, Quinones-Hinojosa A. Association of surgically acquired motor and language deficits on overall survival after resection of glioblastoma multiforme. Neurosurgery 2009;65(03):463-469; discussion 469-470

6 Chaichana KL, Garzon-Muvdi T, Parker S, et al. Supratentorial glioblastoma multiforme: the role of surgical resection versus biopsy among older patients. Ann Surg Oncol 2011;18(01):239-245

7 Dziurzynski K, Blas-Boria D, Suki D, et al. Butterfly glioblastomas: a retrospective review and qualitative assessment of outcomes. J Neurooncol 2012;109(03):555-563

8 Balaña C, Capellades J, Teixidor P, et al. Clinical course of highgrade glioma patients with a "biopsy-only" surgical approach: a need for individualised treatment. Clin Transl Oncol 2007;9(12): 797-803

9 Chaichana KL, Jusue-Torres I, Lemos AM, et al. The butterfly effect on glioblastoma: is volumetric extent of resection more effective than biopsy for these tumors? J Neurooncol 2014;120(03):625-634

10 Cao L, Li C, Zhang Y, Gui S. Surgical resection of unilateral thalamic tumors in adults: approaches and outcomes. BMC Neurol 2015; 15:229

11 Kelly PJ. Stereotactic biopsy and resection of thalamic astrocytomas. Neurosurgery 1989;25(02):185-194; discussion 194-195

12 Jackson C, Gallia GL, Chaichana KL. Minimally invasive biopsies of deep-seated brain lesions using tubular retractors under exo- scopic visualization. J Neurol Surg A Cent Eur Neurosurg 2017;78 (06):588-594

13 Kelly PJ, Goerss SJ, Kall BA. The stereotaxic retractor in computerassisted stereotaxic microsurgery. Technical note. J Neurosurg 1988;69(02):301-306

14 Labib MA, Shah M, Kassam AB, et al. The safety and feasibility of image-guided BrainPath-mediated transsulcal hematoma evacuation: a multicenter study. Neurosurgery 2017;80(04):515-524

15 Eliyas JK, Glynn R, Kulwin CG, et al. Minimally invasive transsulcal resection of intraventricular and periventricular lesions through a tubular retractor system: multicentric experience and results. World Neurosurg 2016;90:556-564

16 Raza SM, Recinos PF, Avendano J, Adams H, Jallo GI, QuinonesHinojosa A. Minimally invasive trans-portal resection of deep intracranial lesions. Minim Invasive Neurosurg 2011;54(01):5-11

17 Recinos PF, Raza SM, Jallo GI, Recinos VR. Use of a minimally invasive tubular retraction system for deep-seated tumors in pediatric patients. J Neurosurg Pediatr 2011;7(05):516-521

18 Chaichana KL, Cabrera-Aldana EE, Jusue-Torres I, et al. When gross total resection of a glioblastoma is possible, how much resection should be achieved? World Neurosurg 2014;82(1-2):e257-e265

19 McGirt MJ, Chaichana KL, Gathinji M, et al. Independent association of extent of resection with survival in patients with malignant brain astrocytoma. J Neurosurg 2009;110(01):156-162

20 Keles GE, Chang EF, Lamborn KR, et al. Volumetric extent of resection and residual contrast enhancement on initial surgery as predictors of outcome in adult patients with hemispheric anaplastic astrocytoma. J Neurosurg 2006;105(01):34-40

21 Hervey-Jumper SL, Berger MS. Maximizing safe resection of lowand high-grade glioma. J Neurooncol 2016;130(02):269-282

22 Garrett MC, Pouratian N, Liau LM. Use of language mapping to aid in resection of gliomas in eloquent brain regions. Neurosurg Clin N Am 2012;23(03):497-506

23 Keles GE, Lundin DA, Lamborn KR, Chang EF, Ojemann G, Berger MS. Intraoperative subcortical stimulation mapping for hemispherical perirolandic gliomas located within or adjacent to the descending motor pathways: evaluation of morbidity and assessment of functional outcome in 294 patients. J Neurosurg 2004;100(03):369-375

24 Motomura K, Natsume A, Iijima K, et al. Surgical benefits of combined awake craniotomy and intraoperative magnetic resonance imaging for gliomas associated with eloquent areas. J Neurosurg 2017;127(04):790-797

25 Hatiboglu MA, Weinberg JS, Suki D, et al. Impact of intraoperative high-field magnetic resonance imaging guidance on glioma surgery: a prospective volumetric analysis. Neurosurgery 2009;64 (06):1073-1081; discussion 1081

26 Stummer W, Pichlmeier U, Meinel T, Wiestler OD, Zanella F, Reulen HJ; ALA-Glioma Study Group. Fluorescence-guided surgery with 5-aminolevulinic acid for resection of malignant glioma: a randomised controlled multicentre phase III trial. Lancet Oncol 2006;7(05):392-401

27 Andrews RJ, Bringas JR. A review of brain retraction and recommendations for minimizing intraoperative brain injury. Neurosurgery 1993;33(06):1052-1063; discussion 1063-1064

28 Jo KW, Shin HJ, Nam DH, et al. Efficacy of endoport-guided endoscopic resection for deep-seated brain lesions. Neurosurg Rev 2011;34(04):457-463

29 Greenfield JP, Cobb WS, Tsouris AJ, Schwartz TH. Stereotactic minimally invasive tubular retractor system for deep brain lesions. Neurosurgery 2008;63(04, Suppl 2):334-339; discussion 339-340

30 Jho HD, Alfieri A. Endoscopic removal of third ventricular tumors: a technical note. Minim Invasive Neurosurg 2002;45(02):114-119

31 White T, Chakraborty S, Lall R, Fanous AA, Boockvar J, Langer DJ. Frameless stereotactic insertion of Viewsite Brain Access System with microscope-mounted tracking device for resection of deep brain lesions: technical report. Cureus 2017;9(02):e1012 